\title{
Erratum to: Towards automatic bounding box annotations from weakly labeled images
}

\author{
Christian X. Ries $^{1} \cdot$ Fabian Richter ${ }^{1} \cdot$ Rainer Lienhart $^{1}$
}

Published online: 4 July 2015

(C) Springer Science+Business Media New York 2015

\section{Erratum to: Multimed Tools Appl \\ DOI 10.1007/s11042-014-2434-z}

Due to an error during typesetting, the name of author Rainer Lienhart was incorrect in the original version of this article. The correct authors' list is given above. The original version was corrected. Springer apologizes for the mistake. 